\title{
1 Coevolution of male and female reproductive traits drive cascading 2 reinforcement in Drosophila yakuba
}

3

4 Aaron A. Comeault ${ }^{1}$, Aarti Venkat ${ }^{2}$ and Daniel R. Matute ${ }^{1}$

5

6 Biology Department, University of North Carolina, Chapel Hill, North Carolina

7250 Bell Tower Drive, Genome Sciences Building

8 Chapel Hill, NC

9 27510, USA

10

$11{ }^{2}$ Department of Human Genetics

12 The University of Chicago

13920 East 58th Street

14 Chicago, IL

15 60637, USA.

16

17 Correspondence:

18 Biology Department, University of North Carolina, 250 Bell Tower Road, Chapel Hill, 27599.

19 Tel: 919-962-2077

20 Fax: 919-962-1625

21 E-mail: dmatute@email.unc.edu

22

23 Keywords: reinforcement, cryptic sexual choice, experimental evolution.

24 Running title: reinforcement in Drosophila yakuba

25 


\section{ABSTRACT}

When the ranges of two hybridizing species overlap, individuals may 'waste' gametes on

29 inviable or infertile hybrids. In these cases, selection against maladaptive hybridization can lead

30 to the evolution of enhanced reproductive isolation in a process called reinforcement. On the

31 slopes of the African island of São Tomé, Drosophila yakuba and its endemic sister species $D$.

32 santomea have a well-defined hybrid zone. Drosophila yakuba females from within this zone

33 show increased postmating-prezygotic isolation towards $D$. santomea males when compared

34 with D. yakuba females from allopatric populations. To understand why reinforced gametic

35 isolation is confined to areas of secondary contact and has not spread throughout the entire $D$.

36 yakuba geographic range, we studied the costs of reinforcement in D. yakuba using a

37 combination of natural collections and experimental evolution. We found that $D$. yakuba males

38 from sympatric populations sire fewer progeny than allopatric males when mated to allopatric $D$.

$39 y a k u b a$ females. Our results suggest that the correlated evolution of male and female

40 reproductive traits in sympatric $D$. yakuba have associated costs (i.e., reduced male fertility) that

41 prevent the alleles responsible for enhanced isolation from spreading outside the hybrid zone. 


\section{INTRODUCTION}

Reinforcement is the evolutionary process through which prezygotic reproductive isolation

46 is strengthened by natural selection acting against the production of maladapted, infertile, or

47 inviable hybrids (Dobzhansky 1937, Coyne and Orr 2004, Pfennig and Pfennig 2012).

48 Reinforcement is expected to drive the evolution of prezygotic isolation in regions where two

49 closely related species overlap and hybridize (Dobzhansky 1937, Coyne and Orr 2004). This

50 generates a pattern of "reproductive character displacement," in which individuals of different

51 species found in the same area (sympatry) display greater behavioral isolation from one another

52 than individuals from different areas (allopatry; Brown and Wilson 1956, Rice and Pfenning

53 2008, Pfennig and Pfennig 2009). Reinforcement is abundant and can be found in a wide range

54 of taxonomic groups, including fungi (Anderson et al. 1980, Turner et al. 2010, Murphy and Zeyl

55 2015), animals (Noor 1995, Gerhardt 1994, Pfennig 1998, Rundle and Schluter 1998, Higgie et

56 al. 2000, Haavie et al. 2004, Jaenike et al. 2006), and plants (Kay and Schemske 2008, Hopkins

57 and Rauscher 2011, 2012, Hopkins 2013). Therefore, reinforcement could be common during the

58 'completion' of speciation (Hudson and Price 2014).

59 Data describing specific aspects of the process of reinforcement are becoming

60 widespread: we now have precise measurements of the strength of reinforcing selection (Hopkins

61 et al. 2014), know that reinforcement can occur despite gene flow (Sanderson 1989, Servedio

62 2000, Nosil et al. 2003 Matute 2010b), and have evidence that reinforcement can promote

63 divergence within species, fostering speciation (Liou and Price 1994, Nosil 2005, Pfennig and

64 Rice 2014, Nosil and Hohenloe 2012). Still, important aspects of reinforcement remain

65 unexplored. Particularly we lack information regarding how variation in reproductive isolation is

66 maintained across a species' geographic range. The persistence of different levels of

67 reproductive isolation between populations of a single species in the face of gene flow and

68 stabilizing selection (Sanderson 1989, Servedio and Kirkpatrick 1997, Servedio and Noor 2003)

69 poses a conundrum. One possible explanation is that reinforced reproductive isolation (RRI)

70 favors phenotypes that are selected against in allopatry. In this case, the traits and alleles

71 involved in reinforcement would not spread across a species' geographic range but would remain 
72 confined to areas of secondary contact (Walker 1974, Caisse and Antonovics 1978, Howard

73 1993, Pfennig and Pfennig 2009).

74 Recent work suggests that phenotypes favored by reinforcing selection in sympatry are

75 often disadvantageous in allopatry (Higgie and Blows 2008; Lemmon 2009; Hopkins et al. 2014;

76 Pfennig and Rice 2014; Kozak et al. 2015). These studies provide evidence that reinforcing

77 selection acting in sympatry can affect levels of reproductive isolation observed among

78 conspecific populations: initiating the evolution of reproductive isolation and speciation through

79 a process referred to as 'cascading reinforcement' (Howard 1993; Lemmon 2009; Kozak et al.

80 2015). To date, all reported cases of cascading reinforcement or of fitness costs associated with

81 phenotypes involved in RRI come from studies in which premating reproductive isolating

82 mechanisms are reinforced. For premating reproductive traits, it may be difficult to determine

83 whether reproductive character displacement in sympatry results from indirect selection against

84 the production of hybrids or direct selection on traits involved in species recognition (Shaw and

85 Mendelson 2013).

86 In addition to traits involved in premating isolation, reinforcing selection can act on post-

87 mating traits (Coyne 1974, e.g., gametic isolation: Lee et al. 1995, Wullschleger et al. 2002,

88 Springer and Crespi 2007, Turner et al. 2010, Matute 2010 reviewed in Palumbi 2008).

89 Examples of post-mating traits include proteins involved in interactions between reproductive

90 tracts and gametes (reviewed in Palumbi 2008). Postmating-prezygotic phenomena have

91 physiological and behavioral consequences that can influence both female and male fitness

92 (Pitnick et al 1991, Yapici et al. 1998, Knowles et al. 2004, Markow and O'Grady 2008).

93 Coevolution of these traits can be rapid when there is sexual conflict: ejaculate traits that directly

94 increase male fitness can have deleterious effects on female fitness (Bateman 1948, Fowler and

95 Partridge 1989, Chapman et al. 1995, Arnqvist and Rowe 2002, Wigby et al. 2005, Wigby et al.

96 2009). Previous experiments in Drosophila have demonstrated ongoing coevolution between the

97 female reproductive tract and male ejaculate (Rice 1996, Holland and Rice 1999, Knowles and

98 Markow 2001, Bono et al. 2011, Kelleher et al. 2011, Manier et al. 2013). Traits associated with

99 the female reproductive tract and male ejaculate can therefore become finely tuned within

100 populations (through continual antagonistic coevolution) but not necessarily across 
101 subpopulations within a species (Rice and Hostert 1993, Rice 1996, Manier et al. 2013). This

102 provides a hypothetical mechanism for how RRI that evolves in sympatry could have cascading effects on levels of reproductive isolation among conspecific populations. the evolutionary dynamics of reinforcement in D. yakuba. Drosophila yakuba is a humancommensal species that is widespread throughout sub-Saharan Africa and has extended its range

107 to islands in the Gulf of Guinea. On the volcanic island of São Tomé (off the coast of Cameroon 108 and Gabon), D. yakuba occurs at low elevations (below 1,450 m), and is mostly found in open 109 and semi-dry habitats commonly associated with human settlements (Lemeunier et al. 1986, 110 Matute et al. 2009). In contrast, D. santomea, the sister species of D. yakuba, is endemic to the 111 highlands of São Tomé where it is thought to exclusively breed on endemic figs (Ficus 112 chlamydocarpa fernandesiana; Lachaise et al. 2000, Llopart et al. 2005a, b). The two species 113 come into secondary contact and hybridize in the midlands of the mountain Pico de São Tomé 114 within a well-defined hybrid zone (Llopart et al. 2005a, b). Drosophila yakuba females from this 115 hybrid zone show higher postmating-prezygotic isolation toward males of $D$. santomea than do 116 D. yakuba females from outside the hybrid zone (Matute 2010a), via an unknown mechanism 117 acting within the female reproductive tract (Matute 2010a). There is no indication of RRI in $D$. 118 santomea, even though some genetic variance seems to segregate in this species (Matute 2010b). The RRI observed in sympatric $D$. yakuba can also evolve rapidly in experimental 120 populations derived from allopatric lines (Matute 2010a, b). Matute (2010a, b) imposed strong 121 selection against hybrids in experimental populations where D. yakuba and D. santomea were 122 maintained in sympatry and both behavioral and gametic isolation increase in less than ten 123 generations. These results show that the genetic variation required for behavioral and gametic 124 isolation to evolve is present in allopatric populations. Experimental evolution can thus allow us 125 to test factors that affect the reinforcement of reproductive isolation.

$126 \quad$ Here, we aim to determine whether D. yakuba lines that show RRI in female gametic 127 isolation evolve correlated phenotypes in males, as would be predicted under a scenario of 128 coevolution between female and male traits. In particular, we studied whether male fertility 129 varies across an altitudinal transect spanning the range of $D$. yakuba on Pico de São Tomé. We 
130 show that natural lines of $D$. yakuba where females have RRI towards $D$. santomea males also

131 have lower average male fertility in conspecific matings. Additionally, we show that when

132 enhanced gametic isolation evolves after experimental sympatry with $D$. santomea, lower male

133 fertility also evolves as a correlated trait. For D. yakuba females, enhanced postmating-

134 prezygotic isolation from $D$. santomea males is advantageous in the hybrid zone, but our results

135 suggest that lower male fertility outside of areas of potential interbreeding with $D$. santomea is a

136 correlated cost of reinforced gametic isolation. 


\section{METHODS}

\section{Characterization of the hybrid zone}

140 We collected males from the yakuba species subcomplex (D. yakuba, D. santomea and 141 their reciprocal hybrids) in a transect on the north side of the island of São Tomé starting at sea

142 level and ending at the D. yakuba/D. santomea hybrid zone at 1,200m above the sea level. The

143 collection sites are shown in Supplementary Figure 1. We sampled Drosophila males from 17

144 localities as described in Matute (2015). We counted the number of D. santomea, D. yakuba, and 145 both types of reciprocal $F_{1}$ hybrids. To quantify levels of hybridization at each locality, we 146 qualitatively scored the abdominal pigmentation of all the collected individuals that belonged to 147 the yakuba subcomplex of species. We focused only on male flies, as they are much easier to 148 identify than females. Drosophila yakuba males have a dark abdomen, while $D$. santomea males 149 have a yellow abdomen. $\mathrm{F}_{1}$ hybrid males from the 우 D. yakuba $\times \sigma^{\top}$ D. santomea cross have an 150 intermediate abdominal pigmentation that is different from pure D. yakuba in the A3 and A4 151 segments (Llopart et al. 2002). $\mathrm{F}_{1}$ hybrid males from the reciprocal 우D. santomea $\times \sigma^{\top} D$. $152 y a k u b a$ cross have a patch of brown pigmentation in the A2 and A3 segments (Llopart et al. 153 2002). Counting $F_{1}$ males constitutes a conservative estimate of the production of hybrids as it 154 does not aim to identify individuals from advanced intercrosses.

\section{Stocks}

157 In addition to collecting hybrid males, we established 344 isofemale lines from putative 158 D. yakuba females collected from 17 different locations along the transect. Although Drosophila $159 y a k u b a$, D. santomea, and their hybrids have different colored abdomens, the differences 160 between hybrid females and pure species are subtle (Llopart et al. 2002) and abdomen 161 pigmentation varies within D. yakuba (Matute and Harris 2013). Therefore, we did not use 162 abdominal pigmentation to distinguish pure species from hybrid females. Instead, we took 163 advantage of the fact that pure species $D$. yakuba females produce only sterile males when 164 crossed to D. santomea males (Lachaise et al. 2000) to determine if the collected females, and 165 thus the isofemale lines, were pure species D. yakuba or advanced generation $D$. yakuba 
166 backcrosses. If the crossed females had 'pure' $D$. yakuba genotypes, then they would produce

$167100 \%$ fertile progeny when crossed with $D$. yakuba and $100 \%$ sterile progeny when crossed to $D$.

168 santomea. On the other hand if putatively pure $D$. yakuba were in fact of recent hybrid origin,

169 they would produce sterile male progeny when crossed to either parental (e.g. 90\% if the female

170 is a $F_{1}$ hybrid; see SI for details). Of the 344 collected isofemale lines, we were able to

171 successfully establish 297 isofemale stocks that fulfilled the criteria of being considered 'pure'

172 D. yakuba (SI; Supplementary Table 1).

173 For experimental evolution assays (see below), we generated genetically heterogeneous

174 strains of each species (i.e., synthetic lines) by combining virgin males and females from several

175 isofemale lines collected on São Tomé outside of the hybrid zone (i.e., all lines were from

176 allopatric populations). The synthetic D. santomea line (D. santomea SYN 2005) was generated

177 by J.A. Coyne by combining six isofemale lines collected in 2005 at the field station Bom

178 Sucesso (elevation 1,150 m). The synthetic D. yakuba line (D. yakuba SYN2005) was generated

179 by combining five isofemale lines collected by J.A. Coyne in 2005 on Pico de São Tomé

180 (elevation $880 \mathrm{~m}$ ). All stocks were kept in large numbers after they were created. All rearing was

181 done on standard cornmeal/Karo/agar medium at $24^{\circ} \mathrm{C}$ under a $12 \mathrm{~h} \mathrm{light/dark} \mathrm{cycle.}$

183 Male fertility: sympatry versus allopatry

184 We quantified levels of male fertility by counting the number of progeny produced

185 following crosses between females and males of 20 isofemale lines (all pairwise combinations).

186 To ensure that these lines were unambiguously sympatric or allopatric ten were collected at the

187 low elevation end of the transect described above (low elevation = allopatric lines) while the

188 other 10 were collected in the hybrid zone (high elevation = sympatric lines). These 20 lines

189 showed no evidence for adaptation to different temperatures (temperature covaries with

190 elevation) or the evolution of reproductive isolating mechanisms apart from those described

191 below (see SI for details). Moreover, these lines show only moderate genetic differentiation

192 (median $\mathrm{F}_{\mathrm{ST}}=0.0503$ ) and no evidence of large chromosomal differences (see SI for details).

193 These results indicate that allopatric and sympatric lines differ primarily in whether they

194 occurred in in the absence or presence of $D$. santomea. 
For each of the twenty lines, we collected virgin males and females under $\mathrm{CO}_{2}$ anesthesia and kept them in isolation for 3 days in single-sex groups of 20 flies. On day 4, we conducted no-choice mating trials as previously described (Coyne et al. 2002, Matute and Coyne 2010). Briefly, we combined a single female and a single male, observed whether the pair mated and, if so, recorded copulation latency and copulation duration. Females that showed an abnormally short copulation (<20 minutes) were discarded as no sperm transfer occurs before that time

201 (Chang 2004). After 1 hour, we ended the observations and discarded any females that had not mated. To prevent females from remating, males were removed from each vial by aspiration after mating was finished. Each mated female was allowed to oviposit for $24 \mathrm{~h}$. We then transferred

204 the female to a fresh vial and counted the total number of eggs laid. The counting was repeated 205 daily for 10 days. We scored 10 males per cross for a total of 4,000 males. Crosses were

206 classified as being one of six possible types: 우 allopatric $\times$ ○ $^{\top}$ sympatric, 우 sympatric $\times$

207 万allopatric, 우 allopatric $\times \sigma^{\nearrow}$ allopatric (different isofemale lines), 우 sympatric $\times \sigma^{\nearrow}$ sympatric

208 (different isofemale lines), 우 allopatric $\times \circlearrowleft^{\top}$ allopatric (within isofemale lines), and 우 sympatric $209 \times \sigma^{\top}$ sympatric (within isofemale lines).

210 We analyzed the data using a generalized linear mixed effect model (GLMM) in which

211 the number of eggs laid by a female (fertility) was the response, the cross type was the fixed

212 effect (the six cross types defined above), and the identity of the isofemale lines were random

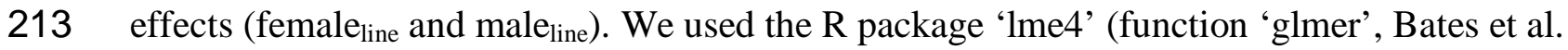

214 2011) and fitted the model, assuming Poisson distributed error, with the form:

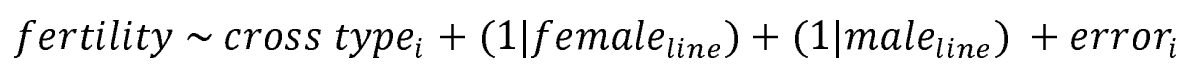

217 To determine whether the type of cross explained a significant proportion of variation in fertility,

218 we compared the proportion of residual deviance explained by the model described above to one

219 lacking the fixed effect of cross type using two methods: 1) a likelihood ratio test ('lrtest'

220 function of the 'Imtest' R package) and 2) parametric bootstrapping ('PBmodcomp' function in

221 the 'pbkrtest' R package, Halekoh and Højsgaard 2014). Parametric bootstrapping was carried

222 out using 100 bootstrapped samples. We also used pairwise Tukey contrasts ('glht' and 'linfct' 
223 functions of the 'multcomp' R package) to determine the types of crosses, if any, differed in

224 level of fertility.

225

226

Geographic distribution of gametic isolation and male fertility

i) Female gametic isolation from D. santomea

When mated to D. santomea males, sympatric $D$. yakuba females (i.e., from the hybrid zone) lay fewer eggs than allopatric females (Matute 2010a). We quantified the geographic scale of female gametic isolation in D. yakuba isofemale lines collected along the altitudinal transect across São Tomé. We used ten lines from each of ten collection locations for a total of 100

234 isofemale lines. Lines were chosen randomly from the 297 pure D. yakuba lines and are listed in 235 Supplementary Table 1. We collected virgin D. yakuba females from each of these 100 isofemale 236 lines and let them age to four days (as described above) and then mated them to D. santomea 237 SYN2005 males (heterospecific cross). Mated females were kept, and the number of eggs they 238 produced was scored every 24 hours over the course of ten days. In parallel, we mated D. yakuba 239 females from each isofemale line to males from the same isofemale line and counted the number 240 of eggs produced by each female (conspecific cross). Heterospecific and conspecific pairings 241 were monitored in parallel to ensure that mating occurred under the same environmental 242 conditions. For each line, we scored the number of eggs produced by fifteen females in both 243 heterospecific and conspecific matings $(\mathrm{N}=15$ females $\times 100$ lines $=1,500$ females for each type 244 of mating). The proportion of eggs produced after heterospecific matings relative to conspecific 245 matings was taken as an inverse proxy for the magnitude of gametic isolation (i.e., the more eggs 246 produced after a single heterospecific mating the weaker the gametic isolation; Chang 2004).

To analyze whether there were differences in the strength of gametic isolation between 248 isofemale lines collected from sympatric versus allopatric regions, we fitted GLMMs with 249 Poisson distributed error using the "glmer" function in the "lme4" R package. We treated the 250 number of eggs produced in heterospecific and conspecific crosses as separate data sets. The two 251 GLMMs were therefore constructed as: 


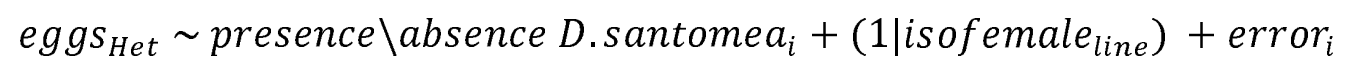

and

produced using both maximum likelihood tests (LRT, 1 degree of freedom) and parametric

259 bootstrapping (100 bootstrap samples, function 'PBmodcomp' in the 'pbkrtest' R package;

260 Halekoh and Højsgaard 2014) that compared the two models to two 'null' models that lacked the

261 fixed effect of presence $\backslash$ absence of $D$. santomea.

262

ii) Intraspecific male fertility

We next quantified male fertility along the altitudinal transect using the same $100 \mathrm{D}$.

$266 y a k u b a$ isofemale lines used to study female gametic isolation. We crossed males from each of

267 these lines with conspecific females from two tester stocks: one allopatric and one sympatric.

268 Drosophila yakuba Täi18 (hereafter referred to as allopatric ${ }_{\text {Täi18), }}$, is an allopatric isofemale line,

269 collected in the Täi forest on the border between Liberia and Ivory Coast. BOSU1250.5

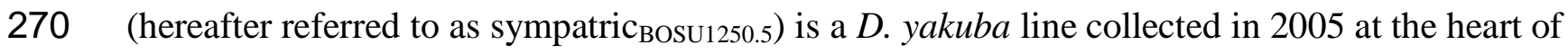

271 the São Tomé hybrid zone and is considered sympatric. We collected virgin males and when they

272 were 4 days old, allowed them to mate to virgin females from either tester stock following the

273 mating procedure described above (no-choice trials). The number of eggs produced over ten days

274 was assessed as a proxy for male fertility with females from different populations. Crosses with

275 allopatric $_{\text {Täi18 }}$ and sympatric ${ }_{\mathrm{BOSU} 1250.5}$ were considered different datasets. Each dataset was

276 analyzed by fitting a GLMM with Poison distributed error where the number of eggs produced

277 per cross was the response, the origin of the male (whether the isofemale line is sympatric or

278 allopatric) was the fixed effect, and isofemale line was a random effect: 


$$
\text { fertility } \sim \text { presence } \backslash \text { absence D. santomea } a_{i}+\left(1 \mid \text { isofemale }_{\text {line }}\right)+\text { error }_{i}
$$

For both 'allopatric' and 'sympatric' data sets, the model described above was compared with a model without the fixed effect of presence $\backslash$ absence of $D$. santomea using both maximum likelihood tests (LRT, 1 degree of freedom) and parametric bootstrapping (100 bootstrap samples, function 'PBmodcomp' in the 'pbkrtest' R package; Halekoh and Højsgaard 2014).

\section{Correlation between gametic isolation and male fertility}

To determine whether the average magnitude of gametic isolation from $D$. santomea males and average male fertility per isofemale line were correlated across isofemale lines, we used Spearman's rank correlation tests implemented in the 'stats' package in R (function 'cor.test'). Bonferroni correlation tests gave similar results.

Previous work has shown that strong selection against hybridization can drive the 296 evolution of enhanced gametic isolation in female D. yakuba that are kept in experimental 297 sympatry with $D$. santomea (Matute 2010a,b). If reinforced gametic isolation and reduced male 298 fertility are correlated traits, they should evolve together in experimental sympatry. We took an

299 experimental evolution approach to test this hypothesis and establish whether lower male fertility 300 between allopatric and sympatric populations of $D$. yakuba could evolve as a correlated trait with 301 enhanced gametic isolation from $D$. santomea observed in females.

303 allopatric population (D. yakuba SYN2005) in experimental sympatry with $D$. santomea (D.

304 santomea SYN2005) for ten generations following previously described experimental protocols

305 (Koopman 1950, Higgie et al. 2000, Blows and Higgie 2002, Matute 2010a). Each bottle contained 250 D. yakuba females, 250 D. yakuba males, 250 D. santomea females, and 250 D. 
307 santomea males. To set up each successive generation, we collected 250 flies of each sex of $D$.

308 yakuba (identifiable by their abdominal pigmentation) as virgins from the experimental bottles

309 and transferred them into new bottles. To reconstitute sympatric conditions, $250 \mathrm{D}$. santomea

310 flies of each sex (collected as virgins from stock bottles) were added to each bottle. All flies were

311 collected between seven and ten hours after eclosion once the flies had already achieved their

312 adult pigmentation. Hybrids were recognized by their abdominal pigmentation (intermediate -

313 yet different - between the two parental species) and were discarded. This procedure was

314 followed for ten generations. Twenty-three control populations (i.e. bottles) of $D$. yakuba were

315 maintained in parallel with the same number of conspecifics (500 flies per bottle) but in the

316 absence of $D$. santomea. The maintenance conditions and population size of $D$. yakuba were the

317 same between the experimental sympatry and control bottles. The strength of gametic isolation

318 was measured after ten generations of experimental sympatry using methods described

319 previously (see "Geographic distribution of gametic isolation and male fertility" above).

320 We compared levels of female gametic isolation from $D$. santomea, male fertility with

321 sympatric BOSU1250.5 females, and male fertility with allopatric $_{\text {Täi18 }}$ females after 10 generations of

322 experimental sympatry. We fitted generalized linear models (GLMs) with Poisson distributed

323 error (i.e., Poisson regression) in which the magnitude of gametic isolation or male fertility was

324 the response and the generation (0, or 10) was the fixed effect. To assess the affect of time (i.e.,

325 generation) on the evolution of gametic isolation or male fertility we used likelihood ratio tests

326 comparing models including versus excluding this term. Models were fitted using the 'glm'

327 function in R. Finally, we used Spearman's rank correlation to compare whether there was a

328 correlated response in levels of isolation from $D$. santomea observed in females and isolation

329 from allopatric D. yakuba observed in males across experimental lines. 


\section{RESULTS}

\section{Characterization of the hybrid zone}

We sampled from across the hybrid zone between D. santomea and D. yakuba on the island of Pico de São Tomé to measure the distributions of D. santomea, D. yakuba, and their two reciprocal hybrids. The abundances of each of the four genotypes ( 2 pure species and two $\mathrm{F}_{1}$

338 hybrids) at 17 locations along the altitudinal transect is shown in Figure 1. Hybrids were found in 339 the same locations as four years prior (Llopart et al. 2005a), suggesting that the hybrid zone has 340 remained stable since it was first reported. Notably we found a few D. santomea males at $700 \mathrm{~m}$

341 indicating that $D$. santomea males sometimes wander out of the canonical distribution previously 342 reported for the species.

344 Male fertility: Sympatry versus allopatry

We have previously described the reproductive advantage obtained by $D$. yakuba females

347 that evolved RRI both in nature, and under experimental evolution (Matute 2010a, 2010b). We

348 hypothesized that there may be an associated cost to RRI that prevents it from spreading to

349 allopatric populations of D. yakuba. We mated males and females of allopatric and sympatric

350 lines in all 4 possible combinations, and counted how many eggs were produced by each mating

351 type. We found that males from sympatric areas sire fewer progeny than allopatric males when

352 mated to allopatric females but not when mated to sympatric females (Figure 2b). Generalized

353 linear mixed models fitted to the data showed that the type of cross being conducted (the fixed

354 effect in these models) explained a significant proportion of residual deviance in the number of

355 eggs produced when compared to a null model lacking this term (LRT: $\chi^{2}=700.09$, df $=5, P<1$

$356 \times 10^{-15}$; parametric bootstrapping: $P=0.01$; Supplementary Table 3 ). Pairwise comparisons

357 revealed that the average number of eggs laid in $\sigma^{7}$ sympatric $\mathrm{x}$ 우 allopatric crosses $(\mathrm{mean}=$

35875.38 , standard error $[\mathrm{SE}]=0.21)$ was significantly lower compared to the number of eggs laid

359 in all other cross types (range of mean [and SE] across other types of cross $=88.79-90.23[0.28$

$360-0.91])$; all $P<0.0001$; Figure 1; Supplementary Table 4). 


\section{Geographic distribution of gametic isolation and male fertility}

We characterized the geographic distribution of RRI along an altitudinal transect on Pico de São Tomé by collecting 10 D. yakuba isofemale lines at each of 10 different sites along the transect ( $\mathrm{N}=100$, including the 20 lines we used above) and measuring the strength of gametic isolation in $D$. yakuba females towards $D$. santomea males. We focused on lines that were putatively pure $D$. yakuba (rather than lines with potentially admixed ancestry; see SI). We fitted GLMMs using sympatry with $D$. santomea as a fixed effect and isofemale line as a random effect. We found that the magnitude of gametic isolation between populations of $D$. yakuba and $D$. santomea is affected by sympatry with $D$. santomea (LRT: $\chi^{2}=54.37, \mathrm{df}=1, P=1.66 \times 10^{-}$

372 13 ; parametric bootstrapping: $P=0.0099$; Figure 3a, black boxes), conforming the expectation

373 that enhanced gametic isolation is driven by maladaptive hybridization with $D$. santomea.

374 Female fertility in conspecific matings also differed between allopatric and sympatric lines

375 (LRT: $\chi^{2}=86.97, \mathrm{df}=1, P<1 \times 10^{-15}$; parametric bootstrapping: $P=0.0099$; Figure 3a, grey

376 boxes); however, the magnitude of the difference in mean number of eggs produced between

377 sympatric and allopatric D. yakuba females was much lower in conspecific crosses (mean eggs

$378[\mathrm{SE}]$ : sympatric females $=86.70$ [0.17]; allopatric females $=93.19$ [0.18]) when compared to

379 heterospecific crosses (mean eggs [SE]: sympatric females = 34.60 [0.47]; allopatric females = $38063.35[0.60])$.

We next asked whether male fertility varied among the same lines collected along the

382 transect. We measured male fertility by mating males from each isofemale line to either

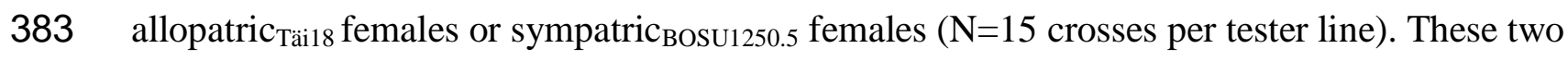

384 lines had previously been identified as representative sympatric and allopatric lines (Matute

385 2010a). The results from these crosses are shown in Figure 3b. We fitted two GLMMs similar to

386 those used to study the magnitude of gametic isolation observed in females: one for matings with

387 sympatric $_{\mathrm{BOSU} 1250.5}$ females and one for matings with allopatric Täi18 $_{18}$ females. Male fertility did not

388 change between regions of sympatry and allopatry when mated to sympatric ${ }_{\mathrm{BO} O \mathrm{SU} 1250.5}$ females

389 (LRT: $\chi^{2}=86.97, \mathrm{df}=1, P=1 \times 10^{-15}$; parametric bootstrapping: $P=0.0099$; Figure $3 \mathrm{~b}$, grey 
boxes). By contrast, male fertility differed between allopatric and sympatric regions when males were mated to allopatric Täil $_{18}$ females (LRT: $\chi^{2}=0.2144, \mathrm{df}=1, P=0.64$; parametric bootstrapping: $P=0.63$; Figure $3 \mathrm{~b}$, black boxes). The number of eggs produced by allopatric females suggests that male fertility is lowest in lines that are sympatric with $D$. santomea (Figure $3 b$ ) and that the geographic distribution of reduced male fertility on São Tomé mirrors that of enhanced gametic isolation.

\section{Correlation between gametic isolation and male fertility}

We also examined whether the magnitude of female gametic isolation was correlated with male fertility in crosses made with allopatric ${ }_{\text {Täi18 }}$ and sympatric ${ }_{\text {BOSU1250.5 }_{5}}$ females. We 401 evaluated the correlation between the average strength of gametic isolation and average male 402 fertility when mated with allopatric and sympatric females using 25 females and 25 males per 403 isofemale line. Female gametic isolation was correlated with male fertility with allopatric Taiils $_{\text {B }}$

404 females (Spearman's rho $=0.885, P<1 \times 10^{-10}$; Figure 4 a) but not with sympatric ${ }_{\text {BOsu1250.5 }}$

405 females (Spearman's rho $=0.116, P=0.250$; Figure $4 \mathrm{~b}$ ). These results illustrate that female 406 gametic isolation toward heterospecifics in sympatry might evolve at the cost of reduced male 407 fertility in conspecific allopatric matings.

\section{Experimental Sympatry}

412 isolation from D. santomea males when evolved under sympatric conditions (Matute 2010a, b).

413 We repeated this experiment and again found that D. yakuba females laid fewer eggs when 414 mated to heterospecific $D$. santomea males following 10 generations of sympatry than were laid 415 at generation 0 (Poisson GLM, LRT: $\chi^{2}=2767.7 ; \mathrm{df}=1 ; P<1 \times 10^{-10}$, Figure 5a). The number of 416 eggs laid following conspecific matings remained the same (LRT: $\chi^{2}=2.43 ; \mathrm{df}=1 ; P=0.12$; 417 Figure 5a). We next looked at whether reduced conspecific male fertility with both allopatric ${ }_{T a ̈ 18}$ 418 and sympatric ${ }_{\mathrm{BOSU} 1250.5} D$. yakuba evolved as a correlated trait with increased female gametic 
419 isolation from $D$. santomea. We found no difference in male fertility among the experimentally

420 evolved lines when mated to sympatric ${ }_{\mathrm{BOSU}} 250.5$ females $\left(\mathrm{LRT}: \chi^{2}=1.06 ; \mathrm{df}=1 ; P=0.304\right.$;

421 Figure $5 b)$. However, male fertility in matings with allopatric Täi18 $_{18}$ females showed a significant

422 decrease over ten generations of experimental sympatry (LRT: $\chi^{2}=743.9$; $\mathrm{df}=1 ; P<1 \times 10^{-10}$,

423 Figure 5b). In control populations of $D$. yakuba raised in parallel to the experimental populations

424 but with $D$. santomea absent, we observed no change in female gametic isolation (LRT: $\chi^{2}=$

$4250.54 ; \mathrm{df}=1 ; P=0.462)$, or male fertility (LRT: $\left.\chi^{2}=0.89 ; \mathrm{df}=1 ; P=0.347\right)$ over the same 10

426 generations of evolution. Finally, we observed a significant correlation between levels of female

427 gametic isolation from $D$. santomea and male fertility with allopatric Täi18 $_{\text {females across }}$

428 experimental replicates (Spearman's rho $=0.69 ; P<1 \times 10^{-10}$; Figure $5 \mathrm{c}$ ) but not with male

429 fertility in matings with sympatric ${ }_{\mathrm{BO} O} 1250.5$ females (Spearman's rho $\left.=-0.046, P=0.115\right)$. These

430 results provide experimental evidence that when gametic isolation from $D$. santomea males

431 evolves via reinforcing selection acting on $D$. yakuba females, there is a correlated cost of

432 postmating prezygotic incompatibility between sympatric males and conspecific females from

433 allopatric populations. 


\section{DISCUSSION}

We have used observation and experimental evidence to show that the reinforcement of gametic isolation between $D$. yakuba females and $D$. santomea leads to the correlated evolution

442 of intraspecific postmating prezygotic isolation as the results of reduced conspecific male

443 fertility. Our results suggest that there is 'fine-tuning' between male and female reproductive

444 traits within populations of D. yakuba. Furthermore, we describe a cost associated with RRI in

445 regions of sympatry with $D$. santomea: when sympatric $D$. yakuba males mate with allopatric $D$.

$446 y a k u b a$ females, they suffer from decreased fertility. Our finding provides a potential causal

447 mechanism for the localized effect of reinforcing selection on reproductive isolation: alleles that

448 underlie female traits that are adaptive in sympatry are associated with alleles that underlie male

449 traits that are maladaptive in geographic regions outside of hybrid zones.

450 Although enhanced gametic isolation in females is highly beneficial in the presence of $D$.

451 santomea (by reducing maladaptive hybridization) it is associated with conditionally lower male

452 fertility. This correlated cost, coupled with the fact that enhanced gametic isolation provides no

453 fitness benefit outside the hybrid zone, likely hampers the spread of the alleles underlying

454 enhanced RRI into allopatry. Our results shed light on why the observed reinforced gametic

455 isolation in the D. yakuba/D. santomea hybrid zone is confined to areas of secondary contact.

456 Together, these results provide evidence for cascading reinforcement among populations of $D$.

$457 y a k u b a$ on the island of São Tomé.

Alternate explanations for observed patterns of reproductive isolation:

Reinforced reproductive isolation is one means by which natural selection can lead to the 461 completion of speciation (Dobzhansky 1937, Lukhtanov et al 1995, Coyne and Orr 2004).

462 However, it has remained unclear how the signature of reinforcement in hybrid zones persists 463 despite migration between allopatric and sympatric populations (Walker 1974, Liou and Price 464 1994, Servedio and Noor 2003). Strong phenotypic differentiation between sympatric and 465 allopatric populations can be generated through a variety of processes that do not involve 466 selection against 'reinforcing' alleles in allopatric regions. For example, local adaptation to 
467 different environments, geographical structuring of populations, drift, and/or genetic

468 incompatibilities could all contribute to reductions in gene flow among conspecific populations

469 and lead to the incidental evolution of reproductive isolation. For the populations and lines of $D$.

$470 y a k u b a$ we focused on in this study, we addressed these issues and found it unlikely that

471 phenotypic differentiation between allopatric and sympatric populations results from $i$ ) local

472 adaptation to temperature, $i$ ) forms of reproductive isolation other than male fertility, iii) strong

473 genetic differentiation among populations at alternate ends of the altitudinal transect, or $i v$ )

474 chromosomal inversions limiting genomic admixture between demes (Hoffman et al. 2004,

475 Kirkpatrick and Barton 2006) (see SI for details). These results are not surprising given that the

476 collection sites for the hybrid zone and the allopatric areas are separated by less than $10 \mathrm{~km}$; a

477 distance some Drosophila species can travel overnight (Jones et al. 1981, Coyne et al. 1982,

478 Coyne and Milstead 1987 but see Timofeef-Ressovsky and Timofeef-Ressovsky 1940; reviewed

479 in Powell 1997). The fact that we observe some non-zero genetic differentiation among

480 isofemale lines derived from females sample from opposite ends of the altitudinal transect

481 suggests that reinforcing selection acting in sympatry - and selection acting in allopatry - could

482 help drive genetic differentiation among conspecific populations: a supposition that warrants

483 further investigation.

485 Fitness tradeoffs and the cost of reinforcement:

The reduced fertility we observed when sympatric males mated to allopatric females

487 represents a fitness tradeoff between selection acting in regions where $D$. yakuba occurs in

488 sympatry with $D$. santomea and selection acting in allopatry. Reduced male fertility also implies

489 indirect costs to females with enhanced gametic isolation in sympatry because their sons will be

490 less fit than those of allopatric females. The tradeoffs could explain why reinforced gametic

491 isolation has not spread throughout the whole geographic range of D. yakuba on São Tomé. Our

492 results add to the growing list of examples of traits favored through reinforcement in regions of

493 sympatry but selected against in allopatry (Lemmon 2009; Hopkins and Rausher 2014; Pfennig

494 and Rice 2014; Kozak et al. 2015). We do not mean to imply that reduced male fertility is the

495 only cost associated with enhanced gametic isolation in D. yakuba females from the hybrid zone. 
496 It is possible that unexplored traits also hamper the spread of enhanced gametic isolation

497 between sympatric and allopatric regions.

499 Coevolution of female and male reproductive traits:

500 At the phenotypic level, our findings highlight the tight link between the female

501 reproductive tract and male ejaculate. In the case of $D$. yakuba we present here, male

502 performance depends on the genotype of his mate (Figures 2, 3, 4 and 5). The evolution of

503 gametic isolation towards D. santomea in female D. yakuba leads to a co-evolutionary change in

504 male traits (manifested as reduced fertility with allopatric conspecifics). This result suggests that

505 there is selectable genetic variation for female and male reproductive traits segregating within

506 natural populations; a result seen in previous studies (Pitnick and Miller 2000, Miller and Pitnick

507 2002). A model of coevolution between male and female reproductive traits in $D$. yakuba would

508 suggest that reinforcing selection can trigger changes in female traits that reduce the production

509 of maladaptive heterospecific offspring, which in turn leads to coevolutionary changes in

510 conspecific male traits.

511 Several lines of evidence indicate that the traits involved in the interactions between the

512 ejaculate and the female reproductive tract are constantly diverging both between and within

513 species (Manier et al. 2013). First, comparative studies have revealed correlated evolution of

514 sperm and female reproductive tract morphology in Drosophila (Pitnick et al. 1997, 1999).

515 Specifically, across the whole genus Drosophila, sperm length (among other sperm traits) is

516 highly variable (Joly and Bressac 1994), and there is a strong positive relationship across species

517 between the length of sperm and the length of the seminal receptacle, the main sperm-storage

518 organ in the female (Pitnick et al. 1999). Furthermore, experimental evolution of $D$.

519 melanogaster in the laboratory has revealed that increased seminal receptacle length can drive

520 the evolution of increased sperm length (Pitnick and Miller 2000, Miller and Pitnick 2002). Our

521 combination of comparative and experimental results suggest that coevolution between the sexes

522 can drive correlated evolution between sperm and female reproductive tract traits, with evolution

523 in female traits resulting in selection on corresponding traits in males. 
At the molecular scale, components of the seminal fluid (such as the accessory gland

525 proteins, Acps), are known to evolve faster than the rest of the genome (Ram and Wolfner 2007).

526 Approximately $36 \%$ of Acps shared among members of the D. melanogaster subgroup ( $D$.

527 melanogaster, D. simulans, D. sechellia, D. erecta, and D. yakuba) appear to have evolved under

528 the influence of positive selection (Swanson et al. 2001a; Mueller et al. 2005; Haerty et al. 2007).

529 Additional studies have shown that Acps evolve rapidly within species (Begun et al. 2000; Kern

530 et al. 2004; Begun and Lindfors 2005; Schully and Hellberg 2006). Such accelerated rates of

531 molecular evolution are not unique to male proteins but have also been observed in genes related

532 to fertilization and female receptors (Andres and Anrqvist 2001, Swanson et al. 2004, Lawniczak

533 and Begun 2007, Chow et al. 2010). These results suggest that Acps and female receptors, the

534 molecular underpinnings of male $\times$ female post-mating interactions, are prone to rapid evolution

535 and species harbor sufficient genetic variation in these traits for natural and sexual selection to

536 act upon.

538 Caveats and conclusions:

539 Our results come with at least three caveats. First, it is possible that sympatric females are

540 of hybrid ancestry. We assessed this issue by measuring sterility of offspring when females were

541 crossed to either D. yakuba and D. santomea (see SI for details). However, this assay will only

542 reveal 'hybrid' D. yakuba lines that contain alleles that are involved in hybrid sterility with $D$.

543 santomea; it is possible that other parts of the genome have admixed ancestry, although it is

544 unclear how this might affect male fertility.

545 A second caveat comes from the population cage sizes used for experimental evolution. It

546 has been shown that high population densities reduce fitness and influence behavior in a variety

547 of organisms (e.g., Booth 1995, Zachar and Neiman 2013, Matute 2014). We chose to have

548 cages with the same number of D. yakuba individuals, to control for levels of genetic variance

549 between treatments; however, we did not explore the evolution of reproductive isolation at

550 different experimental densities.

551 Thirdly, it is worth noting that even though reduced male fertility and enhanced gametic

552 isolation are correlated traits, we have no evidence that these traits have the same genetic basis or 
553 that the alleles controlling the two traits are genetically linked. Since female receptivity $\times$ male

554 ejaculate interactions are finely tuned, changes in female phenotypes, such as sperm retention,

555 might cause concomitant changes in male traits related to ejaculate quality without the need to

556 invoke genetic linkage. Future work using crosses will need to be done to further elucidate this

557 connection.

558 It has long been argued that the evolution of reproductive isolation could be constrained

559 by sexual selection, and that pleiotropic effects of reproductive isolation would either accelerate

560 or hinder speciation (reviewed in Panhuis et al. 2001, Ritchie 2007). Our data demonstrate that

561 the evolution of reinforcement is not free of associated costs, and that sexual selection might

562 oppose reinforcing selection during speciation (Ritchie 2007, Safran et al. 2013). Other cases

563 have been identified in which reinforcing selection leads to the evolution of traits unfavored by

564 sexual selection (Spea multiplicata: Pfennig 1998; Drosophila serrata: Higgie et al. 2000).

565 Finally, our results contribute to the body of work demonstrating that locally adaptive

566 traits can have unexpected costs that might hamper their expansion across the entire range of a

567 species (Littlejohn and Loftus-Hills 1968; Zouros and D'Entremont 1980; Higgie and Blows

568 2008; Hopkins et al. 2014; Pfennig and Rice 2014; Kozak et al. 2015). When a trait contributes

569 to reproductive isolation, the pattern of enhanced reproductive isolation in sympatry that is the

570 hallmark of reinforcement can be explained by selection against the phenotypes involved outside

571 of the hybrid zone. 


\section{FIGURES}

574

575

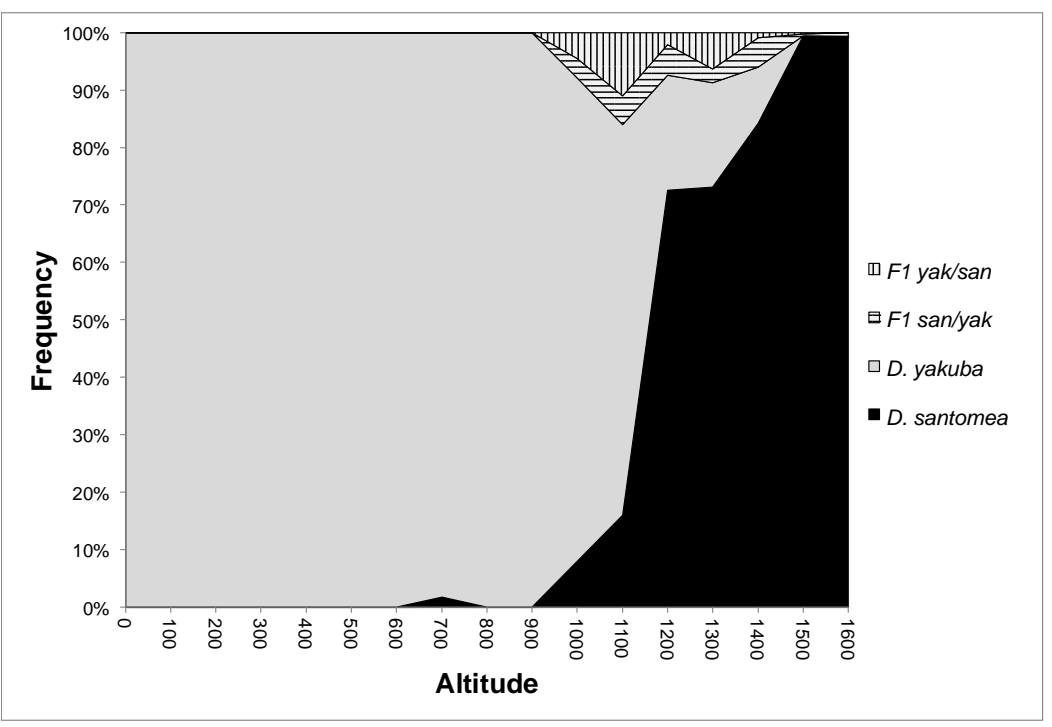

577 FIGURE 1. Abundance of D. yakuba, D. santomea and their $\mathrm{F}_{1}$ hybrids along the

578 altitudinal gradient on Pico de São Tomé. Results presented are for $F_{1}$ males that were

579 identified based on their abdominal pigmentation. 


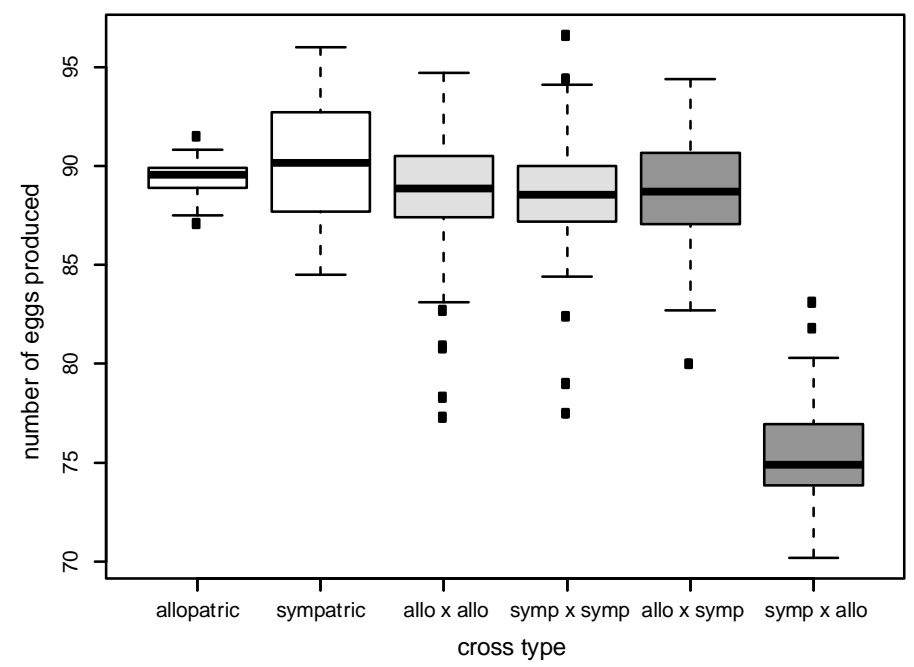

582 FIGURE 2. Males from a sympatric population show reduced fertility when mated to

583 allopatric females. We crossed males and females from 20 isofemale lines in all possible

584 combinations and counted the number of eggs produced over ten days Crosses were split into six

585 categories depending on the identity of the female and the male involved in the cross. The y-axis

586 shows the average number of eggs produced after 10 days across crosses in each category.

587 Categories of crosses are (from left to right): males and females from the same allopatric

588 isofemale line (white box), males and females from the same sympatric isofemale line (white

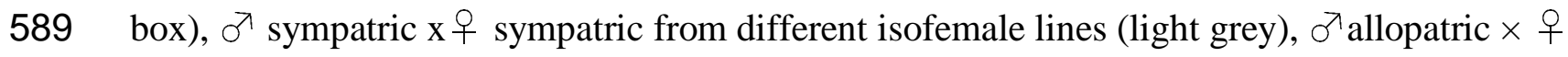

590 allopatric from different isofemale lines (light grey), o $\sigma^{\top 7}$ allopatric $\times$ 우 sympatric lines (dark

591 grey), and $\sigma^{7}$ sympatric $\times$ 우 allopatric lines (dark grey) lines. Sympatric males produced fewer

592 progeny when mated to allopatric females than any of the other possible crosses. 
a) female gametic isolation

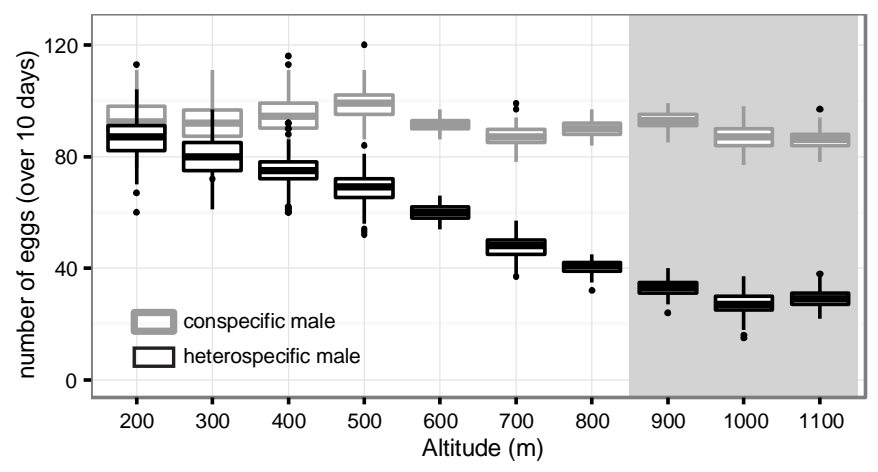

b) male fertility

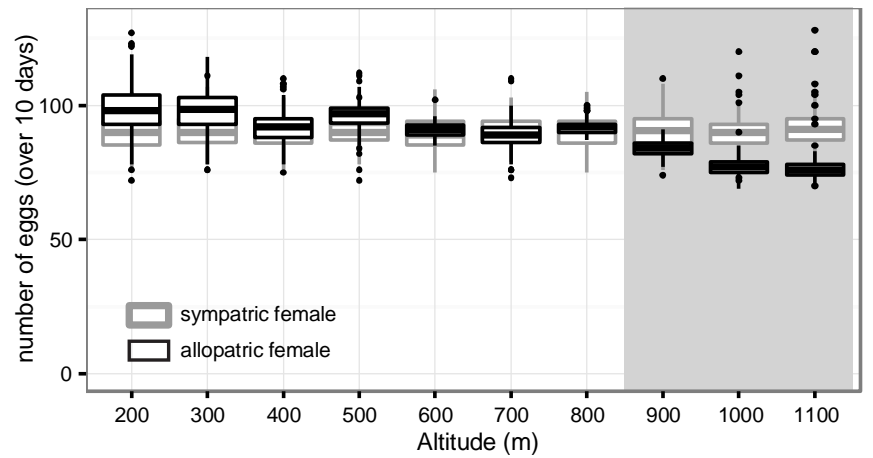

595 FIGURE 3. Levels of gametic isolation and male fertility across the altitudinal transect. We

596 measured the levels of gametic isolation and male fertility in 100 lines collected along the

597 altitudinal transect in São Tomé (10 lines per elevation / site). Gray shaded polygons to the right

598 of each panel demarcate the location of the hybrid zone. a) There is no gametic isolation among

599 females when crossed to D. yakuba males (conspecific crosses; gray boxes); however, isofemale

600 lines collected from closer to the hybrid zone have lower fertility (i.e. higher gametic isolation)

601 when crossed to D. santomea (heterospecific crosses, black boxes). b) Male fertility, measured as

602 the number of eggs produced, is lower for isofemale lines collected from the hybrid zone when

603 mated to female D. yakuba from an alloparic isofemale line (black boxes), but not when mated to

604 female D. yakuba from a sympatric isofemale line (gray boxes). Males from sympatric

605 populations showed lower fertility when mated to allopatric females, but not when mated to

606 sympatric females. 
a)

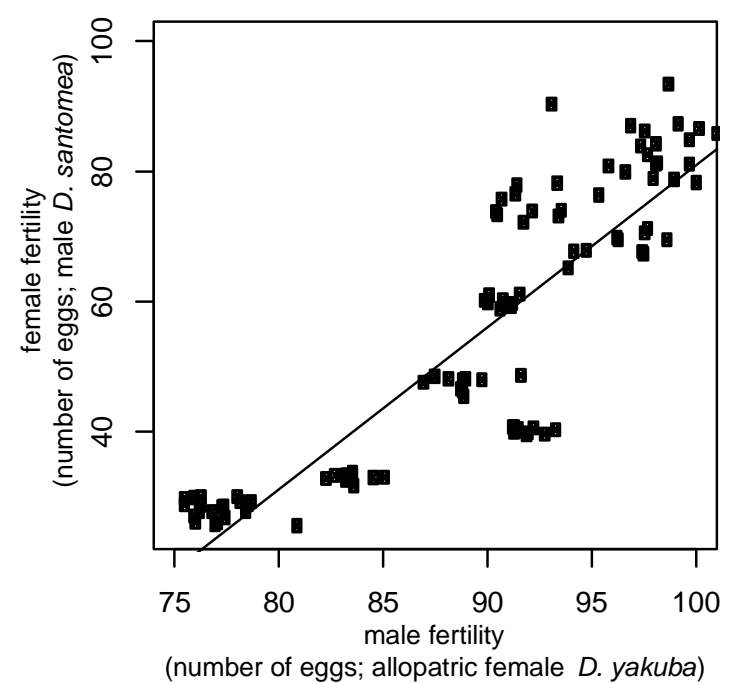

c)

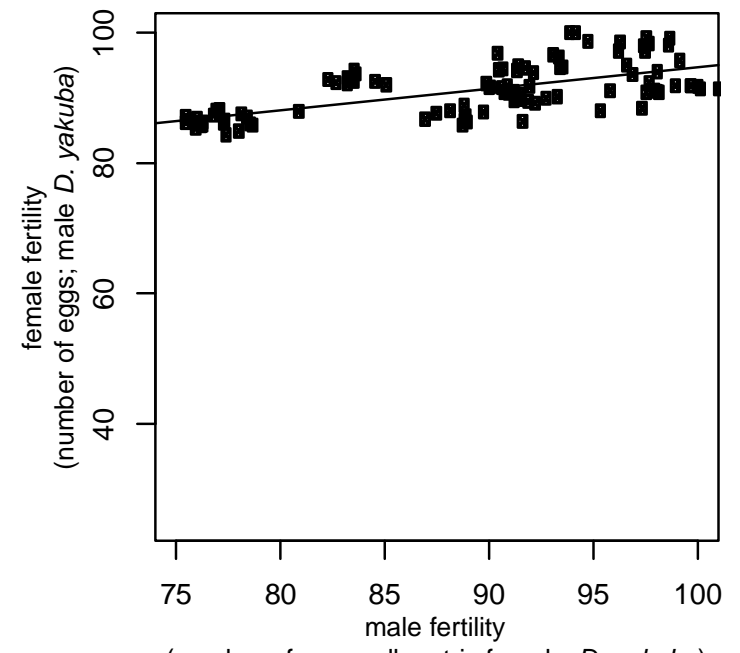

(number of eggs; allopatric female D. yakuba) b)

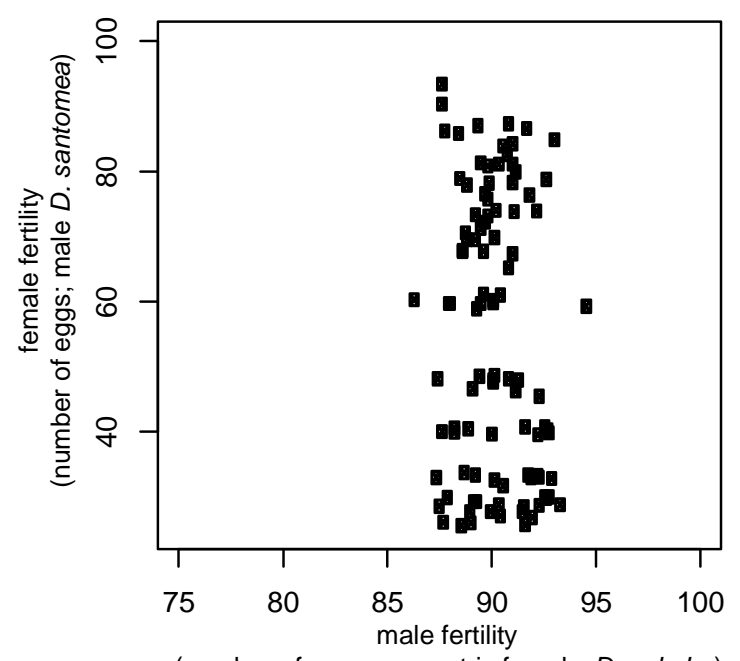

(number of eggs; sympatric female D. yakuba)

d)

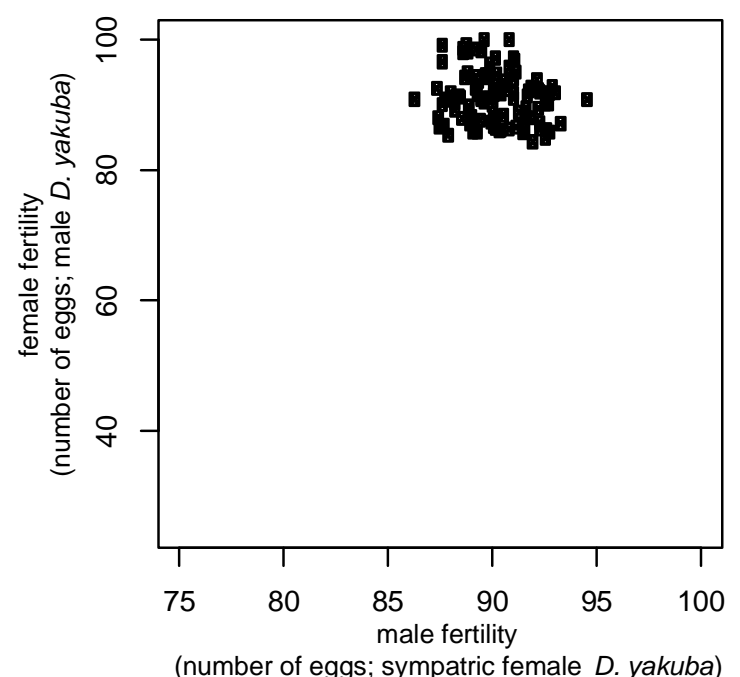

610 populations. a) Male fertility in matings with allopatric females is correlated with the number of

611 eggs laid by females (female gametic isolation) after heterospecific matings with $D$. santomea

612 (as an inverse proxy of the strength of gametic isolation) (Spearman's rho $=0.885, \mathrm{P}<1 \times 10^{-10}$ ).

613 b) Male fertility is not correlated female fertility when mated with sympatric females

614 (Spearman's rho $=0.116, \mathrm{P}=0.250$ ). $\mathrm{c}$ ) The number of eggs laid by females after conspecific 
615 matings is also correlated with male fertility in matings with allopatric females (Spearman's rho $\left.616=0.605, \mathrm{P}<1 \times 10^{-10}\right)$, but not with male fertility in matings with sympatric females $(\mathrm{d}$;

617 Spearman's rho $=-0.149, \mathrm{P}=0.140)$. Lines shown only for significant correlations. 
a) female gametic isolation

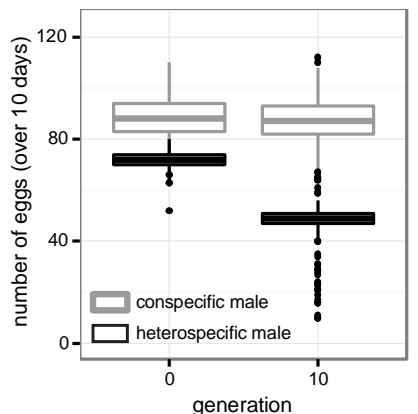

b) male fertility

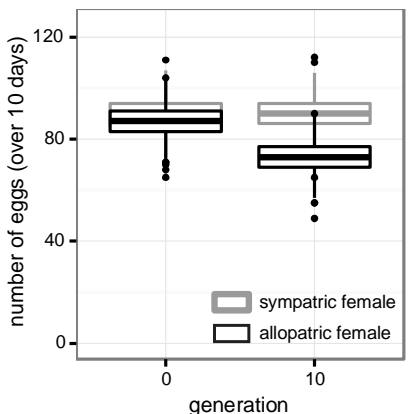

c) correlated evolution of female and male traits

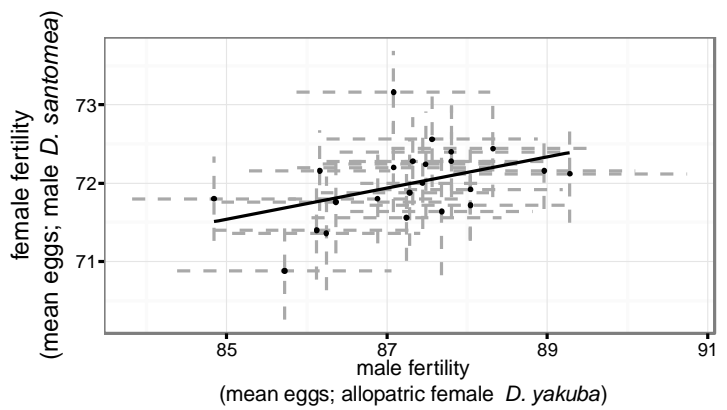

620 FIGURE 5. Experimental evidence that gametic isolation and decreased male fertility

621 coevolve after experimental sympatry with $D$. santomea. a) After 10 generations of

622 experimental sympatry, allopatric $D$. yakuba lines evolved enhanced gametic isolation (black

623 boxes) with no change in conspecific fertility (gray boxes). b) The same evolved lines also

624 showed a decrease in their male fertility when males are mated to an allopatric line of D. yakuba

625 (black boxes) but no decrease in their fertility when mated to females from a sympatric line of $D$.

$626 y a k u b a$ (gray boxes). c) Within experimental populations, the degree of female gametic isolation

627 that evolved over 10 generations ("female fertility"; smaller values represent higher levels of

628 isolation) was correlated with levels of male fertility with allopatric D. yakuba females. Points in

629 "c)" represent means for each of 23 experimental populations and dashed gray lines represent \pm

630 one standard error. Control populations showed no change in gametic isolation from $D$.

631 santomea, female fertility with conspecific males, male fertility when males are mated to

632 allopatric D. yakuba, or male fertility when mated to sympatric D. yakuba (see text for statistics). 


\section{REFERENCES}

Anderson J.B., Korhonen K., Ullrich R.C. (1980) Relationships between European and North American biological species of Armillaria mellea. Experimental Mycology 4: 87-95. Andolfatto, P., Davison, D., Erezyilmaz, D., Hu, T.T., Mast, J., Sunayama-Morita, T., Stern D.L. 2011 Multiplexed Shotgun Genotyping for Rapid and Efficient Genetic Mapping. Genome Res. 21: 610-617. system in houseflies: the footprints of sexually antagonistic coevolution? Proc. R. Soc. B. 268: 557-563. Arnqvist, G. and L. Rowe. 2002. Antagonistic coevolution between the sexes in a group of insects. Nature 415: 787-789. know? Ann. Rev. Genet. 23: 337-370.

Barton, N., and Keightley P.D. 2002. Understanding quantitative genetic variation. Nat. Rev. Genet. 3: 11-20. Bateman A.J. 1948. Intra-sexual selection in Drosophila. Heredity, 2: 349-368. Bates, D., Maechler, M., and Bolker, B. 2011. lme4: Linear mixed-effects models using S4 classes. R package version 0.999375-39. http://CRAN.R-project.org/package=lme4 population genetics of male accessory gland proteins in Drosophila. Genetics 156: 18791888.

Begun, D. J., and Lindfors, H. A. 2005. Rapid evolution of genomic Acp complement in the melanogaster subgroup of Drosophila. Mol. Biol. Evol. 22: 2010-2021. individual fitness and population demography. Ecology 76: 91-106.

664 Blows, M.W., and Higgie M. 2003. Genetic constraints on the evolution of mate recognition 
under natural selection. Am. Nat. 161: 240-253.

Blows, M.W, and Hoffmann, A.A. 2005 A reassessment of genetic limits to evolutionary change. Ecology 86: 1371-1384. transcriptional changes in the reproductive tracts of con- and heterospecifically mated Drosophila mojavensis females. Proc. Nat. Acad. Sci. U.S.A. 108: 7878-7883. 64. Evolution of reproductive isolation in clinal populations. Heredity 40: 371-384. overlapping ranges. Evolution 58: 781-789. 373: 241-244. interactions underlying variation in reproductive phenotypes of Drosophila. Genetics 186: $1355-1365$. distance migration in Drosophila. Am. Nat. 119: 589-595. D. melanogaster alleles from a Maryland orchard. Am. Nat. 130: 70-82. 281.

690 Coyne, J.A., Kim, S.Y., Chang, A.S., Lachaise, D., and Elwyn, S. 2002. Sexual isolation

691 between two sibling species with overlapping ranges: Drosophila santomea and

692 Drosophila yakuba. Evolution 56: 2424-2434.

693 Coyne, J.A., and Orr, H.A. 2004. Speciation. Sunderland, Mass., Sinauer Associates. 
694 Dobzhansky, T. 1937. Genetics and the Origin of Species. Columbia Univ. Press, New York.

695 Fowler K., and Partridge L. 1989. A cost of mating in female fruitflies. Nature 338: 760-761.

696 Gavin - Smyth, J., and Matute, D. R. 2013. Embryonic lethality leads to hybrid male

697 inviability in hybrids between Drosophila melanogaster and D. santomea. Ecol. Evol.,

698 3: $1580-1589$.

699 Gerhardt, H.C. 1994. Reproductive character displacement of female mate choice in the grey

$700 \quad$ treefrog, Hyla chrysoscelis. Anim. Behav. 47: 959-969.

701 Haavie, J., Borge, T., Bures, S., Garamszegi, L.Z., Lampe, H.M., Moreno, J., Qvarnström,

702 A., Török, J., and Sætre, G.P. 2004. Flycatcher song in allopatry and sympatry-

703 convergence, divergence and reinforcement. J. Evol. Biol. 17: 227-237.

704

Haerty, W., Jagadeeshan, S., Kulathinal, R. J., Wong, A., Ram, K. R., Sirot, L. K., Levesque,

705

L., Artieri, C.G., Wolfner, M.F., Civetta, A. and Singh, R. S. 2007. Evolution in the fast

706 lane: rapidly evolving sex-related genes in Drosophila. Genetics 177: 1321-1335.

707

Halekoh, U., and Højsgaard, S. 2014. A Kenward-Roger Approximation and Parametric Bootstrap Methods for Tests in Linear Mixed Models - The R Package pbkrtest. J. Stat. Softw. 59: 1-30.

Higgie, M., Chenoweth, S., and Blows, M.W. 2000 Natural selection and the reinforcement of mate recognition. Science 290: 519-520.

Higgie, M., and Blows M.W. 2007. Are traits that experience reinforcement also under sexual selection? Am. Nat. 170: 409-420.

Higgie, M., and Blows, M.W. 2008. The evolution of reproductive character displacement conflicts with how sexual selection operates within a species. Evolution 62: 1192-1203.

Higgie, M., Chenoweth, S., and Blows, M. W. 2000. Natural selection and the reinforcement of mate recognition. Science 290: 519-521. male attractiveness. Proc. Natl. Acad. Sci. USA 108: 3659-3664.

Hoffmann, A.A., Sgro, C.M., and Weeks, A.R. 2004. Chromosomal inversion polymorphisms and adaptation. Trends Ecol. Evol. 19: 482-488.

722 Holland, B., and Rice, W. R. 1999. Experimental removal of sexual selection reverses 

Sci. USA 96: 5083-5088.

Hopkins, R., and Rausher, M.D. 2011. Identification of two genes causing reinforcement in the Texas wildflower, Phlox drummondii. Nature 469: 411-415.

Hopkins, R., and Rausher, M. D. 2012. Pollinator-mediated selection on flower color allele drives reinforcement. Science 335: 1090-1092. Selection in a Texas Wildflower. Curr. Biol. 24: 1995-1999

Howard, D.J. 1993. Reinforcement: origin, dynamics, and fate of an evolutionary hypothesis, in Hybrid zones and the evolutionary process (Harrison, R. G., ed.), pp.46-69, New York: Oxford University Press.

Hudson, R.R., Slatkin, M., and Maddison, W.P. 1992. Estimation of levels of gene flow from DNA sequence data. Genetics 132: 583-589.

Hudson, E. J., and Price, T. D. 2014. Pervasive reinforcement and the role of sexual selection in biological speciation. J. Heredity 105: 821-833.

Jaenike, J., Dyer, K.A., Cornish, C., and Minhas, M.S. 2006 Asymmetrical reinforcement and Wolbachia infection in Drosophila. PLoS Biol 4: e325.

Joly, D., and Bressac, C. 1994. Sperm length in Drosophilidae (Diptera): estimation by testis and receptacle lengths. International Journal of Insect Morphology and Embryology 23: $85-92$.

Jones, J.S., Bryant, S.H., Lewontin, R.C., Moore, J.A., and Prout, T. 1981. Gene flow and the geographical distribution of a molecular polymorphism in Drosophila pseudoobscura. Genetics 98: 157-178.

Kay, K.M., and Schemske, D.W. 2008. Natural selection reinforces speciation in a radiation of Neotropical rainforest plants. Evolution 62: 2628-2642. suggests sexual conflict in geographically isolated races of Drosophila mojavensis. 
Kern, A. D., Jones, C. D., and Begun, D. J. 2004. Molecular population genetics of male accessory gland proteins in the Drosophila simulans complex. Genetics 167: 725-735.

Kirkpatrick, M., and Barton, N. 2006. Chromosome inversions, local adaptation, and speciation. Genetics 173: 419-434.

Knowles, L.L., Hernandez, B.B., and Markow, T.A. 2004. Exploring the consequences of postmating-prezygotic interactions between the sexes. Proc. R. Soc. Lond. B 271: S357S359.

Koopman, K.F. 1950. Natural selection for reproductive isolation between Drosophila pseudoobscura and Drosophila persimilis. Evolution 4: 135-148. Behavioral isolation due to cascade reinforcement in Lucania killifish. Am. Nat. 185: 491-506.

Lachaise, D., Harry, M., Solignac, M., Lemeunier, F., Bénassi, V., and Cariou, M.L. 2000. Evolutionary novelties in islands: Drosophila santomea, a new melanogaster sister species from São Tomé. Proc. R. Soc. Lond. B. 267: 1487-1495.

Lawniczak M.K., and Begun, D.J. 2007. Molecular population genetics of female-expressed mating-induced serine proteases in Drosophila melanogaster. Mol Biol Evol 24: 19441951.

Lee, Y.H., Ota, T., and Vacquier, V.D. 1995. Positive selection is a general phenomenon in the evolution of abalone sperm lysin. Mol. Biol. Evol. 12: 231-238. group, In The Genetics and Biology of Drosophila, Vol. 3, edited by Ashburner M., Carson, H.L., Thompson, J.N. Academic Press, New York, NY, USA. drives multidimensional reproductive character displacement in frogs. Evolution 63:1155-1170. is not the only possible outcome of reinforcement. J. Evol. Biol. 17: 177-183.

780 Levin, D. A., and Kerster, H. W. 1967. Natural selection for reproductive isolation in Phlox. 
Evolution 21: 679-687.

Levin, D. A., and Schaal, B. A. 1970. Corolla color as an inhibitor of interspecific hybridization in Phlox. Am. Nat. 104: 273-283. Transform. Bioinformatics 25: 1754-1760.

Li, H., Handsaker, B., Wysoker, A., Fennell, T., Ruan, J., Homer, N., Marth, G., Abecasis, G., Durbin, R. and the 1000 Genome Project Data Processing Subgroup. 2009. The Sequence alignment/map (SAM) format and SAMtools. Bioinformatics 25, 2078-2079.

Liou, L.W. and Price, T.D. 1994. Speciation by reinforcement of premating isolation. Evolution 48: 1451-1459.

Littlejohn, M. J., and Loftus-Hills, J. J. 1968. An experimental evaluation of premating isolation in the Hyla ewingi complex (Anura: Hylidae). Evolution 22: 659-663.

795 Llopart, A., Lachaise, D., Coyne, J.A. 2005b. Multilocus analysis of introgression between two sympatric sister species of Drosophila: Drosophila yakuba and D. santomea. Genetics 171: 197-210.

Lukhtanov, V. A., Kandul, N. P., Plotkin, J. B., Dantchenko, A. V., Haig, D., and Pierce, N. E. 2005. Reinforcement of pre-zygotic isolation and karyotype evolution in Agrodiaetus butterflies. Nature 436: 385-389.

801 Manier M.K., Belote, J.M., Lüpold, S., Berben, K.S., Ala-Honkola, O., Collins, W.F., and Pitnick, S. 2013. Rapid diversification of sperm precedence traits and processes among three sibling Drosophila species. Evolution 67: 2348-2362.

804 Markow, T.A., and O'Grady, P. 2008. Reproductive ecology of Drosophila. Functional 805 Ecology 22: 747-759.

806 Matute, D.R., Novak, C.J., Coyne, J.A. 2009 Temperature-based extrinsic reproductive 807 isolation in two species of Drosophila. Evolution 63: 595-612.

808 Matute, D.R. 2010a Reinforcement of gametic isolation in Drosophila. PLoS Biol. 8:

809 e1000341.


810 Matute, D.R. 2010b Reinforcement can overcome gene flow during speciation in Drosophila. Curr Biol 20: 2229-2233. species of Drosophila. Evolution 64: 903-920.

814 Matute, D. R. 2014. The magnitude of behavioral isolation is affected by characteristics of 815 the mating community. Ecology and Evolution, 4: 2945-2956.

816 Matute, D.R. 2015. Noisy neighbors can hamper the evolution of reproductive isolation by 817 reinforcing selection. Am. Nat. 185: 253-269.

818 Miller, G. T. and Pitnick S. 2002. Sperm-female co-evolution in Drosophila. Science 298: 819 1230-1233.

820 Mueller, J. L., Ram K. R., McGraw L.A., Qazi, M.B., Siggia, E.D., Clark, A.D., Aquadro, C.F., and Wolfner, M.F. 2005. Cross-species comparison of Drosophila male accessory gland protein genes. Genetics 171: 131-143.

823

Murphy, H.A. and Zeyl, C.W. 2015. A potential case of reinforcement in a facultatively sexual unicellular eukaryote. Am. Nat. In press.

Nielsen, R., Korneliussen, T., Albrechtsen, A., Li, Y., and Wang J. 2012. SNP Calling, Genotype Calling, and Sample Allele Frequency Estimation from New-Generation Sequencing Data. PLOS ONE 7: e37558 508.

Nosil, P., Crespi, B.J., and Sandoval, C.P. 2003. Reproductive isolation driven by the combined effects of ecological adaptation and reinforcement. Proc. R. Soc. Lond. B. 270: 1911-1918.

834 Nosil, P., and Hohenlohe, P. A. 2012. Dimensionality of sexual isolation during reinforcement and ecological speciation in Timema cristinae stick insects. Evol. Ecol. $836 \quad$ Res. 14: 467-485.

837 Nosil P. 2005. The role of selection and gene flow in the evolution of sexual isolation in 838 Timema walking-sticks and other Orthopteroids. J. Orthoptera Res. 14: 247-253. 
Palumbi, S. R. 2008. Speciation and the evolution of gamete recognition genes: pattern and process. Heredity 102: 66-76.

Panhuis, T. M., Butlin, R., Zuk, M., and Tregenza, T. 2001. Sexual selection and speciation. Trends Ecol. Evol. 16: 364-371.

Pfennig, K.S. 1998. The evolution of mate choice and the potential for conflict between species and mate-quality recognition. Proc. R. Soc. Lond. B. 265: 1743-1748. conspecific matings. Behav. Ecol. 11: 220-227.

Pfennig, K.S., and Pfennig, D.W. 2005. Character displacement as the "best of a bad situation": fitness trade-offs resulting from selection to minimize resource and mate competition. Evolution 59: 2200-2208.

Pfennig, K.S., and Pfennig, D.W. 2009. Character displacement: ecological and reproductive responses to a common evolutionary problem. Q. Rev. Biol. 84: 253-276.

Pfennig, D. W. and Pfennig, K. S. 2012. Evolution's Wedge: Competition and the Origins of Diversity. University of California Press, Berkeley, CA.

Pfennig, K. S., and Rice, A. M. 2014. Reinforcement generates reproductive isolation between neighbouring conspecific populations of spadefoot toads. Proc. R. Soc. B. 281: 20140949.

Pinheiro, J., and Bates D. 2000. Mixed-effects models in S and S-PLUS. Springer, New York, NY, USA.

Pinheiro, J., Bates, D., DebRoy, S., Sarkar, D., and the R Development Core Team. 2013. nlme: Linear and Nonlinear Mixed Effects Models. R package version 3.1-113. URL: http://cran.r-project.org/web/packages/nlme/index.html

Pitnick S., Markow T.A., and Riedy M.F. 1991. Transfer of ejaculate and incorporation of male-derived substances by females in the nannoptera species group (Diptera: Drosophilidae). Evolution 45: 774-780.

Pitnick, S., Spicer, G.S., and Markow, T.A. 1997. Phylogenetic examination of male ejaculatory donations in Drosophila. Evolution 51: 833-845. 
sperm-storage organs in Drosophila. Evolution 53: 1804-1822.

Pitnick, S., and G. T. Miller. 2000. Correlated response in reproductive and life history traits to selection on testis length in Drosophila hydei. Heredity 84: 416-426. Oxford University Press. New York.

874 Prince, G.J., and Parsons, P.A. 1977. Adaptive behaviour of Drosophila adults in relation to temperature and humidity. Aust. J. Zool. 25: 285-290.

Ram, K. R., and Wolfner, M. F. 2007. Seminal influences: Drosophila Acps and the molecular interplay between males and females during reproduction. Integr. Comp. Biol. 47: 427-445.

Ranz, J. M., Maurin, D., Chan, Y. S., Von Grotthuss, M., Hillier, L. W., Roote, J., Ahsburner, M., Bergman, C. M. (2007). Principles of genome evolution in the Drosophila melanogaster species group. PLoS Biol. 5: e152.

Rice, W. R., and Hostert, E. E. 1993. Laboratory experiments on speciation: what have we learned in 40 years? Evolution 47: 1637-1653.

Rice, W. R. 1996. Sexually antagonistic male adaptation triggered by experimental arrest of female evolution. Nature 381: 232-234. character displacement: Why might invaders generally "win" during character displacement? J. Evol. Biol. 21: 696-704. 102.

Rundle, H.D., and Schluter, D. 1998. Reinforcement of stickleback mate preferences: sympatry breeds contempt. Evolution 52: 200-208.

Rundle, H.D., Chenoweth, S.F., and Blows, M.W. 2009. The diversification of mate preferences by natural and sexual selection. J. Evol. Biol. 22:1608-1615.

Ryan, M.J., and Rand, A.S. 1993. Species recognition and sexual selection as a unitary problem in animal communication. Evolution 47: 647-657. 
Safran, R. J., Scordato, E. S., Symes, L. B., Rodríguez, R. L., and Mendelson, T. C. 2013. Contributions of natural and sexual selection to the evolution of premating reproductive isolation: a research agenda. Trends Ecol. Evol. 28: 643-650

900 Sanderson, N. 1989. Can gene flow prevent reinforcement? Evolution 43: 1223-1235.

901 Schully, S. D., and Hellberg, M. E. 2006. Positive selection on nucleotide substitutions and indels in accessory gland proteins of the Drosophila pseudoobscura subgroup. J. Mol. Evol. 62: 793-802.

Servedio, M.R. and Kirkpatrick, M. 1997. The effects of gene flow on reinforcement. Evolution 51: 1764-1772.

Servedio, M.R. 2000. Reinforcement and the genetics of nonrandom mating. Evolution 54: 21-29.

Servedio, M.R., and Noor, M.A. 2003. The role of reinforcement in speciation: theory and data. Annu. Rev. Ecol. Syst. 34: 339-364.

910 Shaw, K. L., and T. C. Mendelson. 2013. The targets of selection during reinforcement. $J$. Evol. Biol. 26:286-287. http://www.psychologie.uni-freiburg.de/Members/singmann/R/afex hybridizing Mytilus population. Evolution 61: 772-783. Evolutionary EST analysis identifies rapidly evolving male reproductive proteins in Drosophila. Proc. Natl. Acad. Sci. USA. 98: 7375-7379. sequence tag analysis of Drosophila female reproductive tracts identifies genes subjected to positive selection. Genetics 168: 1457-1465.

Timofeef-Ressovsky, H., and Timofeef-Ressovsky, N.W. 1940. Population-genetische Versuche an Drosophila II. Aktionbereiche von Drosophila funebris und Drosophila melanogaster. Zeitschrift für induktive Abstammungs-und Vererbungslehre. 79: 35-44. Tonzetich, J. 2004. Orcein staining and the identification of polytene chromosomes. Methods 
Mol. Biol. 247: 249-56.

Turner, E., Jacobson, D.J., and Taylor, D.J. 2010. Reinforced postmating reproductive isolation barriers in Neurospora, an Ascomycete microfungus. J. Evol. Biol. 23: 16421656.

930 Walker, T.J. 1974. Character displacement and acoustic insects. Am. Zool. 14: 1137-1150.

931 Wigby, S., and Chapman, T. 2005. Sex Peptide causes mating costs in female Drosophila 932 melanogaster. Curr. Biol. 15: 316-321.

933 Wigby, S., Sirot, L. K., Linklater J. R., Buehner N., Calboli F.C.F., Bretman A., Wolfner M.

$934 \quad$ F., and Chapman T. 2009. Seminal fluid protein allocation and male reproductive 935 success. Curr. Biol. 19: 751-757.

936 Wullschleger, E.B., Wiehn, J., and Jokela, J. 2002. Reproductive character displacement

937 between the closely related freshwater snails Lymnaea peregra and L. ovata. Evol. Ecol. $938 \quad$ Res. 4: 247-257.

939 Yapici, N., Kim, Y.J., Ribeiro, C., and Dickson, B.J. 2008. A receptor that mediates the post940 mating switch in Drosophila reproductive behaviour. Nature 451: 33-37.

941 Zachar, N., and Neiman, M. 2013. Profound effects of population density on fitness-related $942 \quad$ traits in an invasive freshwater snail. PLoS One, 8: e80067.

943 Zeileis, A., and Hothorn, T. 2002. Diagnostic checking in regression relationships. R News 2:

944 7-10. URL http://CRAN.R-project.org/doc/Rnews

945 Zouros, E., and d'Entremont, C. J. 1980. Sexual isolation among populations of Drosophila 946 mojavensis: response to pressure from a related species. Evolution, 34: 421-430. 